\title{
AdipoRon: a possible drug for colorectal cancer prevention?
}

\author{
Sara Malih $^{1} \cdot$ Rezvan Najafi ${ }^{1}$
}

Received: 30 June 2015 / Accepted: 6 August 2015 / Published online: 18 August 2015

(C) International Society of Oncology and BioMarkers (ISOBM) 2015

\begin{abstract}
Colorectal cancer (CRC) is in the third place of the most common cancers. Certain risk factors can increase the development of CRC, including diet and inheritance. Several studies have shown that there is a potential link between obesity and CRC. Adipose tissue is known to be a largest endocrine organ in the body, with the ability to produce various cytokines including adiponectin. Two types of adiponectin receptor, AdipoR1 and AdipoR2, have been detected in various cancer tissues such as CRC. There is mounting evidence that AdipoR1 signaling occurs mainly through 5' AMP-activated protein kinase (AMPK) and adiponectin inhibits colorectal cancer cell growth via activation of AMPK, thereby suppression of the mammalian target of rapamycin (mTOR) pathway. Thus, adiponectin replacement-based therapies may represent a novel approach in CRC cell growth inhibition in early stages. AdipoRon is an adiponectin-like synthetic small molecule that activated both adiponectin receptors 1 and 2. We hypothesize that AdipoRon has antiproliferative effects of adiponectin and may suppress the CRC cell growth. With clarification of this drug's role in $\mathrm{CRC}$, it can be used as chemoprevention in patients at risk of developing the disease.
\end{abstract}

Keywords Adiponectin - AdipoRon · Colorectal cancer . Adiponectin receptor $1 \cdot$ Adiponectin receptor 2

Rezvan Najafi

re.najafi@umsha.ac.ir; najafi2535@gmail.com

1 Research Center for Molecular Medicine, Hamadan University of Medical Sciences, Hamadan, Iran

\section{Introduction}

$\mathrm{CRC}$ is the third most common cancer and the second leading cause of cancer death [1]. Its initiation is relevant to multi-faceted interactions between host and environment. Some of the important signaling pathways are dysregulated in CRC, including epidermal growth factor receptor (EGFR)-mediated signaling pathways, Wnt signaling, as well as P53, Notch, and eicosanoid signaling pathways [2]. There is strong evidence that proposes mTOR as a key signaling pathway in several cancers, including CRC. The mTOR pathway elevated activity results in translational dysregulation and also acceleration in $\mathrm{G}_{1}-\mathrm{S}$ phase in colon cancer cell lines [3,4]. Another significant factor in $\mathrm{CRC}$ is the nuclear factor kappa-light-chainenhancer of activated B cells (NF-KB), an important transcription factor. Constitutive activation of NF- $\mathrm{KB}$ has been observed in various malignancies, including CRC, and was included in angiogenesis and tumor growth development. Adiponectin has both stimulatory and inhibitory effects on NF- $\mathrm{KB}$ pathway. Adiponectin can downregulate the inhibitor of NF-KB (IKB) phosphorylation resulting in the NF- $k B$ pathway inhibition. Furthermore, adiponectin has been shown to activate NF- $\mathrm{kB}$ in $\mathrm{C} 2 \mathrm{C} 12$ myocytes. Thus, further studies are required to determine the function of adiponectin in CRC [5, 6]. Impaired regulation of these signaling pathways results in apoptosis elusion, uncontrolled cell progression, induction of genetic instability, and improved metastasis and invasiveness.

Adiponectin, a protein of 244 amino acids and one of the most abundant adipokines, circulates in human plasma in forms of full-length adiponectin (f-adiponectin) or globular adiponectin (g-adiponectin) [7]. Adiponectin receptor has two isoforms: the adiponectin receptor 1 (AdipoR1) and adiponectin receptor 2 (AdipoR2). Adiponectin after binding 
to its receptors can activate several intracellular pathways, including AMPK/mTOR, mitogen-activated protein kinases (MAPK), and phosphoinositide 3-kinase (PI3K)/AKT [7-10].

Obesity often leads to impaired regulation of adiponectin. In obese patients, circulating adiponectin levels are reduced and this may be associated with the increased risk of developing immunological, metabolic, and neoplastic diseases including gastric, colon, prostate, cervix, breast, leukemia, and renal cell carcinoma [7, 9-11].

The pathological features of colorectal tumor are affected by plasma concentrations of adiponectin [12]. The mechanism of tumor suppression by adiponectin is not fully understood yet; however, reports indicate that adiponectin can inhibit the expression of endothelial adhesion molecules, angiogenesis, and hematopoiesis [7]. Adiponectin also inhibited cell growth and induced apoptosis in a dose-dependent manner, both in vivo and in vitro [13]. CRC cell lines treated with adiponectin showed a decrease in phosphorylation of PI3K and Akt. Treating CRC cell lines with adiponectin led to activation of AMPK and suppression of mTOR pathway thus inhibition of cancer cell growth. Moreover, knockdown of adiponectin receptors revealed that adiponectin has a suppressive effect on the proliferation of colon cancer cells $[14,15]$. Through the use of knockout mice lacking adiponectin, increased intestinal polyp formation is observed [16-18]. In a study on Japanese patients, it was shown that decreased levels of circulating adiponectin were correlated with an increase in colonic adenomas [19]. In another study, Otani et al. in 2009 investigated the effects of exogenous administration of adiponectin on intestinal polyp formation in mice with an adiponectin gene point mutation to determine the role of adiponectin in colorectal carcinogenesis. Adiponectin inhibited colorectal adenoma growth in the mentioned mice [20]. This evidence reveals that adiponectin plays a suppressive role in the development of different cancer types and links obesity to carcinogenesis.

Okada-Iwabu et al. published the discovery of orally active, synthetic small-molecule, which both binds and activates adiponectin receptors 1 and 2 . They examined the effect of AdipoRon on insulin resistance, type 2 diabetes, and longevity in obese diabetic mouse models. They observed similar effects to adiponectin via AMPK pathway activation with induction of AMPK phosphorylation and peroxisome proliferatoractivated receptor alpha (PPAR- $\alpha$ ) activation in muscle and the liver. Their survey results demonstrated improvements in insulin resistance and glucose intolerance, and also, lower plasma glucose, increase in fatty acid oxidation, oxidative stress reduction, increase in shortened life expectancy, and decrease in expression of proinflammatory cytokines coding genes such as tumor necrosis factor alpha (TNF- $\alpha$ ) were observed [13].

Recently, another study was done to understand the effect of AdipoRon on post-ischemic myocardial apoptosis using mouse models. The results showed that oral administration of AdipoRon to wild-type mice enhanced cardiac function and attenuated post-ischemic cardiac injury. Furthermore, through AdipoRon treatment, myocardial ischemia/ reperfusion (MI/R)-induced apoptotic cell death showed remarkable improvement in mice deficient of either adiponectin knockout (APNKO) or cardiomyocyte-specific AMPK dominant negative (AMPK-DN) [21].

\section{Conclusion}

Altogether, these studies suggest that adiponectin functions as a suppressive factor at an early step of colorectal carcinogenesis and may have positive effects in the prevention of obesityrelated CRC. Because of difficulties in converting adiponectin to a viable drug form, the application of adiponectin receptor agonist is proposed. The similar effects of AdipoRon to adiponectin have been described in a recent study. No study to date has examined the effect of AdipoRon on CRC cell proliferation. Thus, if the results confirm our hypothesis, the adiponectin receptor agonist, AdipoRon, may be a proper choice in obesity-related CRC chemoprevention.

Conflicts of interest None

\section{References}

1. Haggar FA, Boushey RP. Colorectal cancer epidemiology: incidence, mortality, survival, and risk factors. Clin Colon Rectal Surg. 2009;22(4):191.

2. Wu WK, Wang XJ, Cheng AS, Luo MX, Ng SS, To KF, et al. Dysregulation and crosstalk of cellular signaling pathways in colon carcinogenesis. Crit Rev Oncol Hematol. 2013;86(3):251-77.

3. Weijenberg MP, Hughes LA, Bours MJ, Simons CC, van Engeland $\mathrm{M}$, van den Brandt PA. The mTOR pathway and the role of energy balance throughout life in colorectal cancer etiology and prognosis: unravelling mechanisms through a multidimensional molecular epidemiologic approach. Curr Nutr Rep. 2013;2(1):19-26.

4. Gulhati P, Bowen KA, Liu J, Stevens PD, Rychahou PG, Chen M, et al. mTORC1 and mTORC2 regulate EMT, motility, and metastasis of colorectal cancer via RhoA and Rac1 signaling pathways. Cancer Res. 2011;71(9):3246-56.

5. Sakamoto K, Maeda S, Hikiba Y, Nakagawa H, Hayakawa Y, Shibata W, et al. Constitutive NF-KB activation in colorectal carcinoma plays a key role in angiogenesis, promoting tumor growth. Clin Cancer Res. 2009;15(7):2248-58. doi:10.1158/1078-0432. ccr-08-1383.

6. Nagaraju GP, Aliya S, Alese OB. Role of adiponectin in obesity related gastrointestinal carcinogenesis. Cytokine Growth Factor Rev. 2015;26(1):83-93. http://dx.doi.org/10.1016/j.cytogfr.2014. 06.007 .

7. Habeeb BS, Kitayama J, Nagawa H. Adiponectin supports cell survival in glucose deprivation through enhancement of autophagic response in colorectal cancer cells. Cancer Sci. 2011;102(5):999-1006. 
8. Vetvik KK, Sonerud T, Lindeberg M, Lüders T, Størkson RH, Jonsdottir K, et al. Globular adiponectin and its downstream target genes are up-regulated locally in human colorectal tumors: ex vivo and in vitro studies. Metabolism. 2014;63(5):672-81.

9. Yamauchi T, Iwabu M, Okada-Iwabu M, Kadowaki T. Adiponectin receptors: a review of their structure, function and how they work. Best Pract Res Clin Endocrinol Metab. 2014;28(1):15-23. http:// dx.doi.org/10.1016/j.beem.2013.09.003.

10. Nigro E, Scudiero O, Monaco ML, Palmieri A, Mazzarella G, Costagliola $\mathrm{C}$, et al. New insight into adiponectin role in obesity and obesity-related diseases. BioMed Res Int. 2014;2014.

11. Hiyoshi M, Tsuno NH, Otani K, Kawai K, Nishikawa T, Shuno Y, et al. Adiponectin receptor 2 is negatively associated with lymph node metastasis of colorectal cancer. Oncology letters. 2012;3(4): 756-60.

12. Gialamas SP, Petridou ET, Tseleni-Balafouta S, Spyridopoulos TN, Matsoukis IL, Kondi-Pafiti A, et al. Serum adiponectin levels and tissue expression of adiponectin receptors are associated with risk, stage, and grade of colorectal cancer. Metabolism. 2011;60(11): $1530-8$.

13. Okada-Iwabu M, Yamauchi T, Iwabu M, Honma T, Hamagami K-I, Matsuda K, et al. A small-molecule AdipoR agonist for type 2 diabetes and short life in obesity. Nature. 2013;503(7477):493-9. doi:10.1038/nature12656.

14. Luo Z, Zang M, Guo W. AMPK as a metabolic tumor suppressor: control of metabolism and cell growth. Future Oncol. 2010;6(3): 457-70. doi:10.2217/fon.09.174.
15. Sugiyama M, Takahashi H, Hosono K, Endo H, Kato S, Yoneda K, et al. Adiponectin inhibits colorectal cancer cell growth through the AMPK/mTOR pathway. Int J Oncol. 2009;34(2):339.

16. Fujisawa T, Endo H, Tomimoto A, Sugiyama M, Takahashi H, Saito S, et al. Adiponectin suppresses colorectal carcinogenesis under the high-fat diet condition. Gut. 2008;57(11):1531-8.

17. Mutoh M, Teraoka N, Takasu S, Takahashi M, Onuma K, Yamamoto M, et al. Loss of adiponectin promotes intestinal carcinogenesis in Min and wild-type mice. Gastroenterology. 2011;140(7):2000-8. e2. http://dx.doi.org/10.1053/j.gastro.2011. 02.019 .

18. Saxena A, Chumanevich A, Fletcher E, Larsen B, Lattwein K, Kaur $\mathrm{K}$, et al. Adiponectin deficiency: role in chronic inflammation induced colon cancer. Biochim Biophys Acta (BBA) - Mol Basis Dis. 2012;1822(4):527-36.

19. Obeid S, Hebbard L. Role of adiponectin and its receptors in cancer. Cancer Biol Med. 2012;9(4):213-20. doi:10.7497/j.issn.20953941.2012.04.001.

20. Otani K, Kitayama J, Yasuda K, Nio Y, Iwabu M, Okudaira S, et al. Adiponectin suppresses tumorigenesis in ApcMin/+ mice. Cancer Lett. 2010;288(2):177-82. http://dx.doi.org/10.1016/j.canlet.2009. 06.037 .

21. Zhang Y, Zhao J, Li R, Lau WB, Yuan Y-X, Liang B, et al. AdipoRon, the first orally active adiponectin receptor activator, attenuates postischemic myocardial apoptosis through both AMPK-mediated and AMPK-independent signalings. Am J Physiol Endocrinol Metab. 2015;309(3):E275-E82. doi:10.1152/ ajpendo.00577.2014. 